\title{
Effects of pH, glucose and iron sulfate concentration on the yield of biohydrogen by Clostridium butyricum EB6
}

\begin{abstract}
A local bacterial isolate from palm oil mill effluent (POME) sludge, identified as Clostridium butyricum EB6, was used for biohydrogen production. Optimization of biohydrogen production was performed via statistical analysis, namely response surface methodology(RSM), with respect to $\mathrm{pH}$, glucose and iron concentration. The results show that $\mathrm{pH}$, glucose concentration and iron concentration significantly influenced the biohydrogen gas production individually, interactively and quadratically $(\mathrm{P}<0.05)$. The center composite design (CCD) results indicated that $\mathrm{pH} 5.6,15.7 \mathrm{~g} / \mathrm{L}$ glucose and $0.39 \mathrm{~g} / \mathrm{L}$ FeSO4 were the optimal conditions for biohydrogen production, yielding $2.2 \mathrm{~mol} \mathrm{H} / \mathrm{mol}$ glucose. In confirmation of the experimental model, t-test results showed that curve fitted to the experimental data had a high confidence level, at 95\% with t $1 / 42.225$. Based on the results of this study, optimization of the culture conditions for C. butyricum EB6 significantly increased the production of biohydrogen.
\end{abstract}

Keyword: Biohydrogen; Clostridium butyricum EB6; Microbial; Optimization; Response surface methodology 